\title{
Restriction Enzyme
}

National Human Genome Research Institute (NHGRI)

\section{Source}

National Human Genome Research Institute (NHGRI). Restriction Enzyme.

A restriction enzyme is an enzyme isolated from bacteria that cuts DNA molecules at specific sequences. The isolation of these enzymes was critical to the development of recombinant DNA (rDNA) technology and genetic engineering. 